\title{
Biochemical and transcriptomic analyses of the symbiotic interaction between Cremastra appendiculata and the mycorrhizal fungus Coprinellus disseminatus
}

Yanyan Gao ${ }^{1,2}$, Jun $\mathrm{Ji}^{1,2}$, Yujin Zhang ${ }^{1,2}$, Ningxian Yang ${ }^{1,2}$ and Mingsheng Zhang ${ }^{1,2^{*}}$

\begin{abstract}
Background: Cremastra appendiculata is a rare terrestrial orchid with a high market value as an ornamental and medicinal plant. However, the species depends entirely on fungi for seed germination under natural conditions. In a previous study, we have successfully isolated and identified the mycorrhizal fungus Coprinellus disseminatus which was able to induce the germination of C. appendiculata seeds. We then speculated that $C$. disseminatus may do so by breaking the testa imposed dormancy of the seeds. In this study, biochemical and transcriptomic analyses were used to characterize the germination of $C$. appendiculata seeds, collected at different stages of germination, as affected by C. disseminatus.
\end{abstract}

Results: The lignocellulose in the seeds coat of C. appendiculata was degraded by the mycorrhizal fungus resulting in facilitated absorption of water. The rate of decline in lignin content was 67 and $73 \%$ at 6 and 12 days after sowing, respectively. The water content increased from 13 to $90 \%$ during symbiosis. A total of 15,382 genes showing significantly different levels of expression ( $\log _{2}$ FPKM $\geq 2.0$, Qvalue $\leq 0.05$ ) were successfully identified among all libraries, where the highest number of DEGs was shared between 6 days versus 0 day after symbiotic germination. Gene annotation results suggested that 15 key genes related water-status, such as DHN gene family and Xero 1 were down-regulated. The genes zeaxanthin epoxidase ZEP, 9-cis-epoxycarotenoid dioxygenase NCED3 and $\beta$-carotene hydroxylase involved in the biosynthesis of abscisic acid (ABA) were significantly down-regulated in 6 days as compared to 0 day after symbiotic germination.

Conclusions: This work demonstrates that mycorrhizal fungus $C$. disseminatus can stimulate C. appendiculata seeds germination through a mechanism of breaking the testa imposed dormancy and inducing water absorption of the embryo.

Keywords: Cremastra appendiculata, Coprinellus disseminatus, Lignin degradation, Transcriptome analysis, Seed germination

\footnotetext{
*Correspondence: mszhang@gzu.edu.cn

${ }^{2}$ Key Laboratory of Plant Resources Conservation and Germplasm

Innovation in Mountainous Region (Ministry of Education),

Guiyang 550025, Guizhou, China

Full list of author information is available at the end of the article
}

\begin{abstract}
Background
Orchidaceae is the most species-rich family in the plant kingdom with more than 28,000 known species [9]. Many orchid seeds are tiny dust-like, with lignified seed coats, which mainly depend on compatible mycobionts for germination and subsequent growth and survival of the seedlings $[3,6,12,18,34,45]$. The lignified seed
\end{abstract}

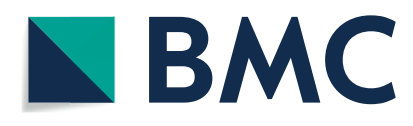

(c) The Author(s) 2022. Open Access This article is licensed under a Creative Commons Attribution 4.0 International License, which permits use, sharing, adaptation, distribution and reproduction in any medium or format, as long as you give appropriate credit to the original author(s) and the source, provide a link to the Creative Commons licence, and indicate if changes were made. The images or other third party material in this article are included in the article's Creative Commons licence, unless indicated otherwise in a credit line to the material. If material is not included in the article's Creative Commons licence and your intended use is not permitted by statutory regulation or exceeds the permitted use, you will need to obtain permission directly from the copyright holder. To view a copy of this licence, visit http://creativecommons.org/licenses/by/4.0/. The Creative Commons Public Domain Dedication waiver (http://creativeco mmons.org/publicdomain/zero/1.0/) applies to the data made available in this article, unless otherwise stated in a credit line to the data. 
coats of the orchids arise mainly from the outer and inner integuments, and contain lignocellulose, suberin, polyphenols and cutin $[4,20,34]$. Lignin is a component of plant cell wall that contains hemicellulose, cellulose and lignin. Lignin binds to cellulose and hemicellulose forming a hydrophobic barrier to water permeation [27, 32]. This barrier enhances the survival of orchid seeds in harsh conditions and enhances wind-aided seed dispersal $[4,12]$. However, the lignified seed coat may interfere with water and nutrient uptake, resulting in 'physically imposed dormancy' [4, 5, 12, 31, 34, 44]. Thus, effectively breaking the testa-imposed dormancy is a critical step in seed germination which a prerequisite for species conservation and large-scale production of orchids.

Orchid mycorrhizal fungi have been reported to be capable of degrading lignin by producing lignin-modifying enzymes and energetically supporting the establishment of orchid seedlings [34, 36, 40]. However, some studies have reported that an efficient enzymatic machinery of mycorrhizal fungi toward lignin was not sufficient for effective break down of lignocellulose in the seed coat, and that some fungi can produce appressorium-like structures or utilize conidia to soften the cell walls [11]. Moreover, the mycorrhizal fungi produce cellulases and pectinases, which contribute to effective penetration of the fungal hyphae into plant tissues [35]. White-rot fungi are recognized as one of the most active microorganisms in lignin degradation. The saprobic fungus Coprinellus disseminatus has been documented to be able to degrade lignocellulose [2,39].

The lignified seed coat, which is thought to be one of the factors in seed dormancy, is formed during the final stages of seed development. Concomitantly, the highly hydrophilic dehydrin $(D H N)$ proteins accumulate in the embryo and serve as protectants against desiccation $[7,10,16,28]$. Dehydrins have multifaceted roles in the protection of plant cells during stress conditions, including drought which triggers ROS accumulation and negatively affects the overall plant performance [37, 48]. Phytohormones strongly regulate seed germination and stress responses. Among which ABA is a primary stress hormone that induces seed dormancy, and regulates stomatal closure to restrict water loss by transpiration [13, $24,49]$. The expression of $D H N$ proteins has also been reported to be induced by both ABA-dependent or ABAindependent mechanisms under drought stress conditions [37].

Cremastra appendiculata, is a rare terrestrial orchid that often grows in the understory of humid and highly shaded forests [45]. It has a high medicinal value because its tubers are effective for cancer treatment, angiogenesis inhibition, heat-clearing, detoxification and detumescence [22, 23, 38, 41]. Due to its commercial and medicinal market value, $C$. appendiculata plant material often illegally collected from the wild populations. In a previous study, we reported that seeds of $C$. appendiculata with intense phenolic compounds in the coats showed enhanced germination and protocorm development in the presence of the mycorrhizal fungus C. disseminatus DJF-10 [14]. However, the mechanisms involved in the symbiotic germination remained unknown.

In this study, seeds at 0 (fungus unpenetrated), 6 (fungus penetrating), 12 (fungus penetrated into embryo and formed pelotons) and 25 days (seeds developed into protocorms) after symbiotic germination were used as test materials to investigate the influence of $C$. disseminatus on the lignocellulose of the seed. We studied the dynamic changes in the lignocellulose content of $C$. appendiculata seeds during symbiotic germination with $C$. disseminatus and then, analyzed the lignocellulose degradation by a combination of methods, including Fourier transform infrared spectroscopy (FTIR), pyrolysis-gas chromatography mass spectrometry (Py-GC/MS) and gas chromatography mass spectrometry (GC-MS). Finally, we analyzed transcriptome of $C$. appendiculata during symbiotic germination, which indicated that $C$. disseminatus possibly degrades lignin in the seed coat, with concomitant increase in water uptake.

\section{Materials and methods}

\section{Experimental materials and symbiotic germination}

The mature seeds of $C$. appendiculata and the mycorrhizal fungus $C$. disseminatus DJF-10 were obtained and stored in the Institute of Plant Physiology and Molecular Biology of Guizhou University, Guiyang city, Guizhou Province, China. The seeds were sterilized with $75 \%$ ethanol (30 s) and then with sodium hypochlorite (1\% effective chloride, $3 \mathrm{~min}$ ). The seeds were germinated in Petri dishes containing oatmeal agar medium (OMA: $4 \mathrm{gL}^{-1}$ rolled oats, $8 \mathrm{gL}^{-1}$ agar, at natural $\mathrm{pH}$ ). Then a $5 \mathrm{~mm}$ plug of actively growing $C$. disseminatus mycelium, precultured on PDA medium, was placed in each OMA Petri dish. The plates were incubated at $22 \pm 2{ }^{\circ} \mathrm{C}$ in full darkness.

\section{Phenotypes of $C$. appendiculata seeds during symbiotic germination with C. disseminatus}

The symbiotically germinated seeds were samples at four stages according to a previous histological study [14]. First, at symbiotic sowing (0 day) (before fungus invasion) where the embryo was surrounded with lignified seed coat. Second, at 6 days (fungus penetrating) where the mycelium penetrated from the suspensor. Third, at 12 days (fungus colonization) where some well-developed pelotons formed and the swollen embryo broke through 
the testa. Fourth at 25 days (pelotons degradation) where more than $70 \%$ of the seeds germinated and formed protocorms with protomeristem at the basal cells were degraded. Therefore, samples were collected at 0 (Fig. 1 0 day), 6 (Fig. 16 days), 12 (Fig. 112 days) and 25 days (Fig. 125 days) after symbiotic sowing (CA, SY1, SY2, and $\mathrm{SY} 3$, respectively) and stored at $-80^{\circ} \mathrm{C}$ for transcriptome research and other subsequent analyses.

\section{Total lignocellulose content and water uptake in C. appendiculata germinating seeds Lignocellulose content}

The dried samples were milled using a pestle and stored in sealed plastic bags. The lignocellulose contents were examined using lignin content assay kit (Solarbio, China). The hemicellulose and cellulose contents were measured using respective assay kits (Sangon Biotech, China) according to manufacturer's instructions.

\section{Water uptake by seeds at different stages of germination}

The symbiotic seeds at different stages of germination were dried at $40^{\circ} \mathrm{C}$ until constant dry weight was reached (48-72 h). The water content was expressed as a percentage of the seed fresh weight for four to five biological replicates.
FTIR and Py-GC/MS analysis of lignocellulose degradation The FTIR was used to follow the degradation of lignocellulose with a Vertex70 (Bruker, Germany) FTIR spectrometer. Before the FTIR, the dried samples were embedded in $\mathrm{KBr}$ pellets at room temperature. FTIR spectrophotometer were recorded in a range of $4000 \mathrm{~cm}^{-1}$ to $400 \mathrm{~cm}^{-1}$. The spectra were recorded in an absorption mode of 16 scans per sample with a resolution of $2 \mathrm{~cm}^{-1}[12,46]$.

The chemical composition of lignocellulose from symbiotic seeds was analyzed by Py-GC/MS according the method of Chen et al. [8] with some modifications. Helium was used as the carrier gas at a constant rate of $1 \mathrm{~mL} \mathrm{~min}^{-1}$. The pyrolysis was carried out at $550^{\circ} \mathrm{C}$, the chromatograph was programmed from 50 to $250^{\circ} \mathrm{C}$ at a rate of $15^{\circ} \mathrm{C} \mathrm{min}^{-1}$ and the final temperature was maintained for $10 \mathrm{~min}$. The chemical compounds were identified by comparing their mass spectra with those at the National Institute of Standards and Technology (NIST) library.

\section{GC-MS analysis of low-molecular-weight degradation products of lignocellulose during symbiotic germination}

To evaluate the lignocellulose degradation efficiency of the mycorrhizal fungus $C$. disseminatus, products from lignocellulose of $C$. appendiculata seeds were analyzed by GC-MS, since this system is appropriate for analysis of the low-molecular-weight compounds. The compounds
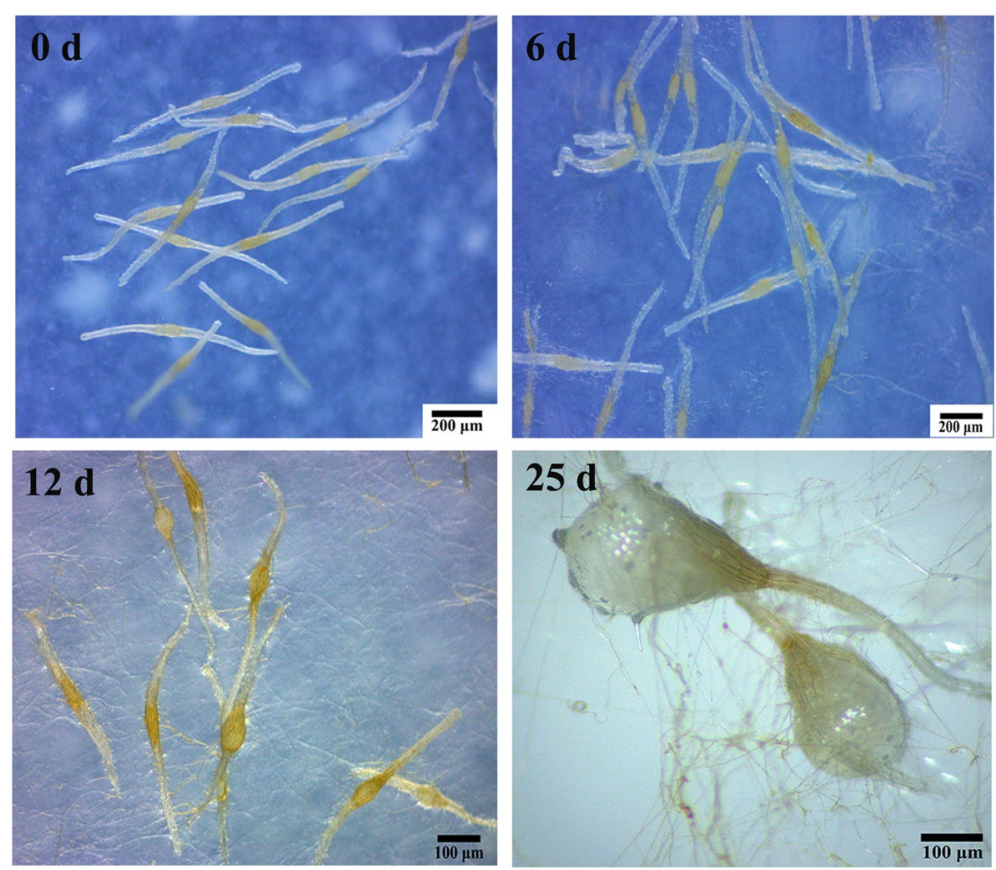

Fig. 1 Phenotypes of the seeds of C. appendiculata symbiotically germinated with C. disseminatus DJF-10 at different stage after sowing. Zero day, six days, twelve days, twenty-five days indicated symbiotic seeds at 0 day, 6 days, 12 days and 25 days after sowing, respectively 
were harvested by crushing the symbiotic medium with a rod and then stirring on shaker at $120 \mathrm{rpm}$ at $10^{\circ} \mathrm{C}$ for $4 \mathrm{~h}$ [2]. After filtering the contents through four layers of cheese cloth, the samples centrifuged at $10,000 \mathrm{rpm}$ for $10 \mathrm{~min}$ at $4{ }^{\circ} \mathrm{C}$. The supernatant was harvested and stored at $-20^{\circ} \mathrm{C}$. The pre-treatment of the supernatant was performed according to Zang et al. [46] with some modifications. The supernatants were acidified with concentrated $\mathrm{HCl}$ to $\mathrm{pH} 2-3$. Then, the samples were extracted three times with ethyl acetate and the organic layers were combined. The combined fractions were dewatered over anhydrous $\mathrm{Na}_{2} \mathrm{SO}_{4}$ passed through a filter paper and evaporated down to $2 \mathrm{~mL}$ at $40^{\circ} \mathrm{C}$. Consequently, $100 \mu \mathrm{L}$ of pyridine and $400 \mu \mathrm{L}$ of $\mathrm{N}$, O-bis (trimethylsilyl) trifluoroacetamide (BSTFA) were added to $500 \mu \mathrm{L}$ samples of the extracted products. The samples were silylated with heating at $60^{\circ} \mathrm{C}$ for $30 \mathrm{~min}$ and filtered through $0.45 \mu \mathrm{m}$ membranes. Aliquots of $1 \mu \mathrm{L}$ of the silylated samples were injected into the GS-MS system. Three replicates were used for each sample.

The GC-MS analysis achieved using a 5975C-7890A system (Agilent, USA) with a DB-5 capillary column $(30 \mathrm{~m} \times 0.25 \mathrm{~mm}, 0.25 \mu \mathrm{m}$ film thickness). In addition, helium was used as the carrier gas at a constant flow rate of $1.0 \mathrm{mLmin}^{-1}$. The column temperature program was set to $60^{\circ} \mathrm{C}(2 \mathrm{~min})$ and $60-260^{\circ} \mathrm{C}\left(10^{\circ} \mathrm{C} \mathrm{min}{ }^{-1}\right.$, hold time of $10 \mathrm{~min}$ ). The injection and detection temperatures were maintained at 200 and $250^{\circ} \mathrm{C}$, respectively. Electron ionization (EI) mass spectra were acquired in full-scan mode from 50 to $55 \mathrm{~m} / \mathrm{z}$. The compounds were identified by comparing their mass spectral data to those in NIST library.

\section{Preparation of RNA for transcriptome analysis}

The four samples of symbiotic seed germination were collected for transcriptome analysis. Additionally, samples of the pure cultured $C$. disseminatus on OMA medium were collected for RNA de novo assembly. Total RNA was extracted from $100 \mathrm{mg}$ using plant RNeasy Kit (Omega Bio-Tek, USA) according to the manufacture's protocol. The extracted RNA was treated with RNase-free DNase I to remove genomic DNA. The quality and quantity of the purified RNA were tested by using NanoDrop One (Nanodrop Technologies Inc., CA, USA), Agilent 2100 Bioanalyzer (Agilent Technologies, Santa Clara, CA, U.S.A.) and $1 \%$ agarose gel electrophoresis. Library construction and sequencing were performed using Illumina HiSeq4000 platform (Illumina Inc., CA, USA) at Beijing Genomics Institute (Shenzhen, China).

\section{De novo assembly of RNA-seq data and functional annotation of unigenes}

The total clean reads were obtained by removing reads containing adapters, ambiguous reads $(N>5 \%)$, and low-quality reads from the raw reads. De novo assembly of the trimmed reads derived from each sample was conducted using Trinity V2.0.6 with default settings. The complete series of fungal and plant transcriptomic data were deposited in the National Center for Biotechnology information (NCBI) database (accession: No. PRJNA762946).

Unfortunately, reference genomes of C. appendiculata and $C$. disseminatus have not been published yet. We were unable to remove the intracellular hyphae from the symbiotic protocorms. Therefore, the fungus $C$. disseminatus de novo group was assembled individually using Trinity at default parameters. The coding sequences of symbiotic protocorms were first mapped to the fungal de novo assembly nonredundant protein (NR) database. Then, all the mapped $C$. disseminatus reads were derived from the symbiotic group $[15,47]$. The remaining unpaired reads were classified as the de novo reference assembly of $C$. appendiculata and were prepared to detect the expression levels. The paired-end reads were mapped to each plant and fungal reference genome assembly using Bowtie2 V2.2.5 [19]. The transcript abundance was estimated using RSEM V1.2.28 [21]. The gene expression levels were estimated by applying the fragments per kilobase per million mapped reads (FPKM). Functional annotation of the unigenes was subjected to NR, Nt, KOG, GO, KEGG, Swissprot and Pfam public databases.

\section{Expression analysis of DEGs}

The differentially expressed genes (DEGs) in library size were identified with DESeq2 and moderated estimation of the fold change $[1,25]$ in the expression level with a fold change $\geq 2.0$ and Qvalue $\leq 0.05$. The GO and KEGG enrichment were performed using the obtained DEGs.

\section{Statistical analysis}

All experiments were repeated at least three times and the results were presented as means $\pm \mathrm{SD}$. The statistical analysis was performed using one-way repeated measures ANOVA, and significant differences were tested by Duncan's test at $p<0.05$.

\section{Results \\ Lignocellulose degradation and water uptake of $C$. appendiculata seeds during symbiotic germination}

To investigate the degradation of lignocellulose by $C$. disseminatus, the loss of lignocellulose dry weight and water absorption during symbiosis were measured. After 6 days of symbiotic germination of $C$. appendiculata with C. disseminatus, the lignin content significantly dropped from $119.48 \mathrm{mg} \mathrm{g}^{-1}$ (CA) to $38.55 \mathrm{mgg}^{-1}$ (SY1) and the lignin degradation rate in the seeds was $73.15 \%$ at 12 days 


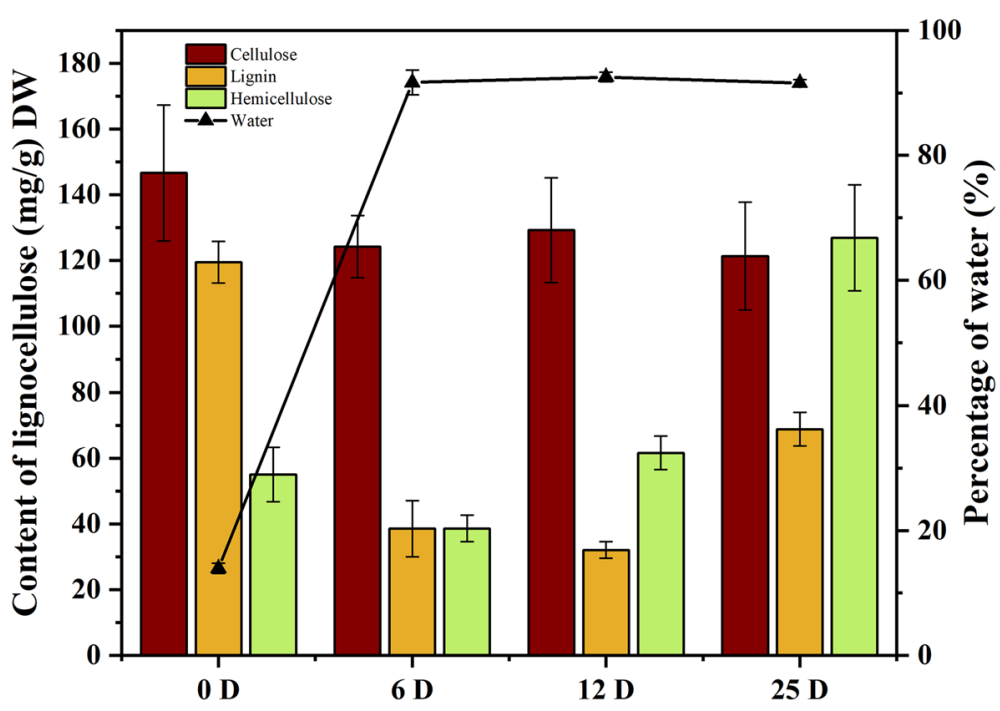

Fig. 2 Lignocellulose content and percentage of water in C. appendiculata seeds during symbiosis. CA, SY1, SY2 and SY3 indicated symbiotic germination at 0 day, 6 days, 12 days, and 25 days after sowing, respectively

(SY2) (Fig. 2). Subsequently, with cell differentiation and protocorm development, lignin accumulation increased to $68.84 \mathrm{mgg}^{-1}$ at SY3, but remain much lower than that of CA. The hemicellulose content was also decreased from $55.04 \mathrm{mgg}^{-1}$ in CA to $38.65 \mathrm{mgg}^{-1}$ in SY1. The hemicellulose degradation rate was $29.78 \%$ in SY1, and significantly increased to $61.61 \mathrm{mgg}^{-1}$ in SY2. With protocorm formation, the hemicellulose content increased to $126.89 \mathrm{mgg}^{-1}$ in SY3. The cellulose content did not change significantly among different stages.

Under symbiosis, the water content increased sharply significantly from CA to SY1 (13.94 and 91.66\%, respectively) and then unchanged at SY2 and SY3 (92.54 and $91.58 \%$, respectively) (Fig. 2). These results revealed that the lignified seed coat was broken by $C$. disseminatus, resulting in enhanced water permeation to the embryo. The results also demonstrate that the seed coat limitation is one of the important components of seed dormancy.

\section{Identification of lignocellulose in symbiotic seeds}

To further validate the lignocellulose degradation in $C$. appendiculata seeds by $C$. disseminatus, the chemical compositions of different samples was characterized by FTIR and Py-GC/MS. The samples showed remarkably different spectra (Fig. 3). Bands of C-lignin in CA were evident at 1650 (strong) and $782 \mathrm{~cm}^{-1}$. Bands of G/S lignin existed at valleys of 1592, 1515 (sharp) and $871 \mathrm{~cm}^{-1}$ (sharp). Under symbiosis with $C$. disseminatus, significant reduction in the lignin signals were recorded at 1650, 1592 and $1515 \mathrm{~cm}^{-1}$ in SY1, SY2 and SY3, respectively as compared to CA. The xylan band of $2923 \mathrm{~cm}^{-1}$ was significantly decreased at SY1, SY2 and
SY3. The absorption band at $1621 \mathrm{~cm}^{-1}$ (arrow 1) may be associated with carbonyl and acetyl groups in xylan degradation intermediates. The band, which was associated with cellulose, disappeared at $1000 \mathrm{~cm}^{-1}$ (arrow 2) during symbiosis. In addition, strong and sharp signals were recorded at 1766 and $1749 \mathrm{~cm}^{-1}$, though it was not clear whether or not they were associated with lignocellulose.

Pyrolysis of the seed symbiotic samples released various families of compounds (Table 1). The fraction of phenolic compounds decreased significantly, while the concentrations furanics and other compounds varied in different samples. These results suggested that the lignocellulose fraction of the seeds was degraded by the symbiotic fungus. In particular, lignin degradation was more significant as demonstrated. Other non-phenolic compounds such as acetic acid and ketone groups resulted from lignin and from the efficient release of phenolic monomers.

\section{GC/MS analysis of low-molecular-weight products} of degradation of lignocellulose of $C$. appendiculata seeds symbiotically germinated with $C$. disseminatus

To identify the products of lignocellulose biodegradation, GC-MS was used to detect the low-molecularweight compounds in OMA symbiotic medium collected at different stages during the symbiotic germination of C. appendiculata seeds with $C$. disseminatus (Table 2; Fig. S1). The degradation products are listed in Table 2. More than 10 degradation products were detected. However, the CA group contained only dibutyl phthalate, suggesting that the compound might have originated from the OMA medium. 


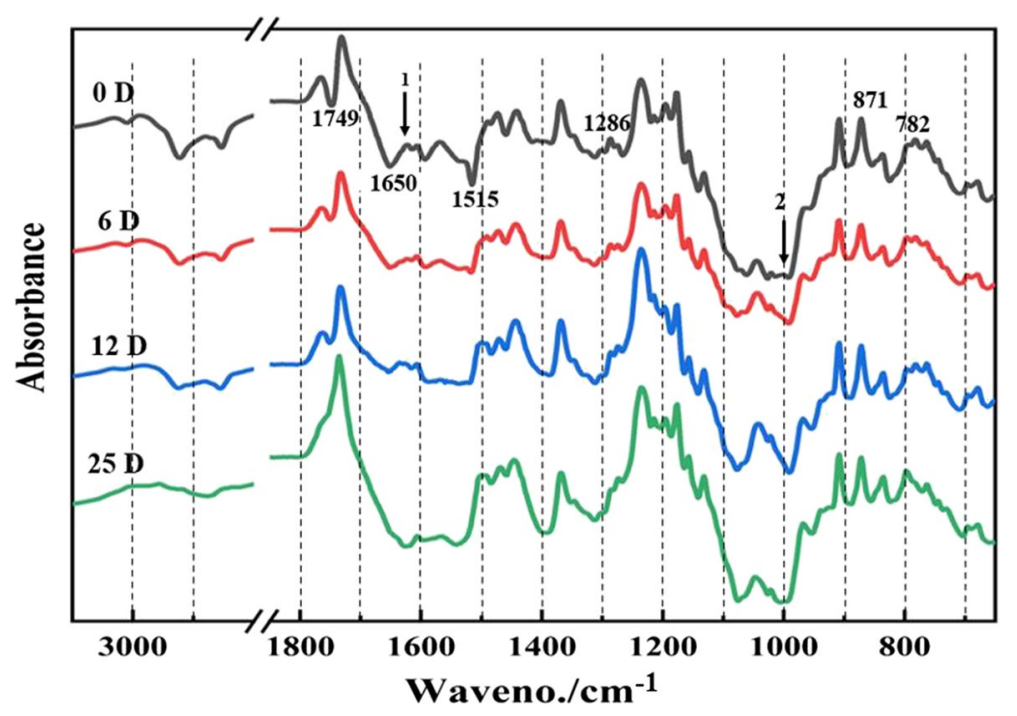

Fig. 3 FTIR spectra of C. appendiculata seeds at different stages of symbiotic germination with C. disseminatus. CA, SY1, SY2 and SY3 indicated symbiotic germination at 0 day, 6 days, 12 days and 25 days after sowing, respectively

Table 1 Part of the chemical compounds released after Py-GC/MS of C. appendiculata seeds and seedings of CA, SY1, SY2 and SY3

\begin{tabular}{|c|c|c|c|c|c|c|c|}
\hline Compound name & MW & Molecular formula & $\begin{array}{l}\text { Retention time } \\
\text { (min) }\end{array}$ & CA & SY1 & SY2 & SY3 \\
\hline Toluene & 92 & $\mathrm{C}_{7} \mathrm{H}_{8}$ & 3.472 & + & + & - & - \\
\hline Cyclobutanol & 72 & $\mathrm{C}_{4} \mathrm{H}_{8} \mathrm{O}$ & 4.247 & + & - & - & - \\
\hline 1-Cyclohexylethylamine & 127 & $\mathrm{C}_{8} \mathrm{H}_{17} \mathrm{~N}$ & 4.422 & + & - & - & - \\
\hline Carbon dioxide & 44 & $\mathrm{CO}_{2}$ & 4.503 & + & - & - & - \\
\hline Phenol & 94 & $\mathrm{C}_{6} \mathrm{H}_{6} \mathrm{O}$ & 6.060 & - & + & + & + \\
\hline 2-Acetyl-5-methylfuran & 124 & $\mathrm{C}_{7} \mathrm{H}_{8} \mathrm{O}_{2}$ & 7.348 & + & - & - & - \\
\hline 4-Ethyl-phenol & 122 & $\mathrm{C}_{8} \mathrm{H}_{10} \mathrm{O}$ & 8.001 & + & + & + & - \\
\hline Isovaleric acid, 3-ethylphenyl ester & 206 & $\mathrm{C}_{13} \mathrm{H}_{18} \mathrm{O}_{2}$ & 8.141 & + & - & - & - \\
\hline 2-Methoxy-5-methylphenol & 138 & $\mathrm{C}_{8} \mathrm{H}_{10} \mathrm{O}_{2}$ & 8.257 & + & - & + & - \\
\hline 4-Ethyl-2-methoxy-phenol & 152 & $\mathrm{C}_{9} \mathrm{H}_{12} \mathrm{O}_{2}$ & 9.114 & + & - & - & - \\
\hline 2-Methoxy-4-vinylphenol & 150 & $\mathrm{C}_{9} \mathrm{H}_{10} \mathrm{O}_{2}$ & 9.458 & + & + & + & - \\
\hline Vanillin & 152 & $\mathrm{C}_{8} \mathrm{H}_{8} \mathrm{O}_{3}$ & 10.361 & + & + & + & - \\
\hline Vanillic acid & 168 & $\mathrm{C}_{8} \mathrm{H}_{8} \mathrm{O}_{4}$ & 10.641 & + & - & - & - \\
\hline Trans-isoeugenol & 164 & $\mathrm{C}_{10} \mathrm{H}_{12} \mathrm{O}_{2}$ & 10.693 & + & + & + & - \\
\hline 1-(3,4-Dimethoxyphenyl)-ethanone & 180 & $\mathrm{C}_{10} \mathrm{H}_{12} \mathrm{O}_{3}$ & 11.644 & + & - & - & - \\
\hline
\end{tabular}

It is well documented that benzene compounds result from degradation of lignin. Five relative compounds (benzeneethanamine, benzene, benzeneacetic acid, benzamide and benzenedicarboxylic acid) were identified in the OMA symbiotic medium of SY1 and SY2. This suggested that the lignin component of the seed coat was degraded, in agreement with FTIR and Py-GC/MS data. Furfural, acetic acid and butanoic acid are degradation products of xylan. Acetic acid was detected in SY1, SY2 and SY3, whereas butanoic acid was detected only in SY2.
Furfural was not detected at any stage. Some other acids and eaters resulting from lignocellulose degradation were detected in SY1 or SY2. These results confirmed that the lignocellulose fraction of the seed coat was degraded by C. disseminatus during symbiotic germination.

\section{RNAseq analysis, de novo assembly, and functional annotation}

Analysis of RNA-seq of C. appendiculata at four developmental stages of symbiotic germination was performed 
Table 2 The chemical composition of lignocellulose degradation products analyzed by GC-MS

\begin{tabular}{|c|c|c|c|c|c|}
\hline Retention time ( $\mathrm{min})$ & Identified compound & CA & SY1 & SY2 & SY3 \\
\hline 8.253 & Benzeneethanamine, N-butyl- $\beta, 4$-bis(trimethylsloxy)- & - & + & + & + \\
\hline 9.693 & Dimethyl adipate & - & - & - & + \\
\hline 10.746 & Benzene, 1,2,3-tris((trimethylsilyl) oxy)- & - & - & + & + \\
\hline 12.980 & Benzeneacetic acid, 4-(trimethylsilyloxy) -3-methoxy & - & - & + & - \\
\hline 12.987 & Ethanedioic acid & - & + & - & - \\
\hline 14.983 & $\begin{array}{l}\text { Phosphoric acid 2-(methoxyimino) ethylbis } \\
\text { (trimethylsilyl) ester }\end{array}$ & - & + & - & - \\
\hline 16.704 & Acetic acid, o-(trimethylsoloxy) phenyl-trimethylsily ester & - & + & + & + \\
\hline 18.213 & Dibutyl phthalate & + & + & + & + \\
\hline 19.652 & Mandelic acid, di(tert-butyldimethylsilyl)- & - & + & - & + \\
\hline 20.911 & Mercaptoacetic acid, bis (trimethylsilyl)- & - & + & + & - \\
\hline 22.105 & Butanoic acid, 2-((trimethylsilyl)oxy-)-, trimethylisly ester & - & - & + & - \\
\hline 22.126 & Benzamide, N-benzyl-N-ethyl-p-isopropyl & - & + & - & - \\
\hline 22.648 & Cis-9-oxabicyclo (6.1.0) no name & - & - & + & - \\
\hline 23.388 & Di (2-ethylhexyl) phthalate & - & + & - & + \\
\hline 25.079 & 1, 2-BenzenediCArboxylic acid, bis (trimethylsilyl) ester & - & + & - & - \\
\hline
\end{tabular}

to characterize the transcriptome changes during seed germination. A total of $41.06 \mathrm{~Gb}$ clean data were generated from each library after filtering out the low-quality data. Among raw reads in all samples, the Q30 values ranged from 91.47 to $92.28 \%$ indicating high-quality reads appropriate for further analysis (Table S1). Since no reference genome was available for C. appendiculata, all $359,510,000$ reads were do novo-assembled into 48,750 CDS with an N50 length of 1398 bp and 97,800 unigenes with an average length of $1120 \mathrm{bp}$ (Table S2). Samples of different biological replicates were clustered separately based on their distinct developmental stages.

For annotation, 97,800 unigenes were subjected to BLASTX search against the sequences in NR, Nt, Pfam, Swissprot, GO, KOG, and KEGG databases (Fig. 4A; Table S3). As a result, a total of 65,911 unigenes (67.39\% of all unigenes) had at least one putative function from one of these databases. For NR annotation, a total of $61,803(63.19 \%)$ unigenes were annotated. As shown in Fig. S2, 36,107 unigenes were assigned with a best score to Dendrobium catenatum (58.42\%) and 14,862 ones were assigned to Phalaenopsis equestris (24.05\%). For GO annotation, 45,555 unigenes were annotated into three GO categories, including cellular component (CC), molecular function (MF), and biological process (BP) (Fig. S3). The cellular component class contained cellular anatomical entity and intracellular and protein-containing complex. The most abundant molecular functions were binding and catalytic activity. Among the biological processes, cellular and metabolic processes were more abundant. Other entries such as biological regulation, localization and response to stimulus were relatively high.
Unigenes among different groups were chiefly classified into carbohydrate transport and metabolism, function unknown, general function prediction only, posttranslational modification, protein turnover, chaperones, signal transduction mechanisms and transcription (Fig. 4B).

\section{Functional annotation of differentially expressed genes Differential expression analysis of gene expression at different stages of symbiotic germination}

To identify the genes involved in symbiosis, we compared, as pairs, the transcriptomes of the four developmental periods of the symbiotic seeds. The pair SY1-CA, represented the symbiotic seeds at 6 days (fungal invasion) compared to 0 day (fungus-free seeds), while SY2SY1 was the symbiotic material at 12 days (fungal colonization) compared to the stage of fungal invasion at day 6 . The pair SY3-SY2 presented the transition to fungal degradation and protocorms with meristem from the fungal colonization at 12 days. A total of 15,382 genes showed significantly different expression levels ( $\log 2$ $F P K M \geq 2.0$, Qvalue $\leq 0.05$ ) among all libraries (Fig. 4C). Among these differentially expressed genes (DEG), 5050 genes were up-regulated and 4818 ones were down-regulated in SY1-CA (Fig. 4D). In SY2-SY1, 5285 genes were up-regulated and 1893 ones were down-regulated in SY2SY1. In SY3-SY2, 449 genes were up-regulated and 669 genes were down-regulated (Fig. 4D; Table S4). We found that the highest number of DEGs was shared between the early mycelium penetration stages (SY1-CA), followed by the early germination stage (SY2-SY1), and then the protocorm stage (SY3-SY2) (Fig. 4D). 

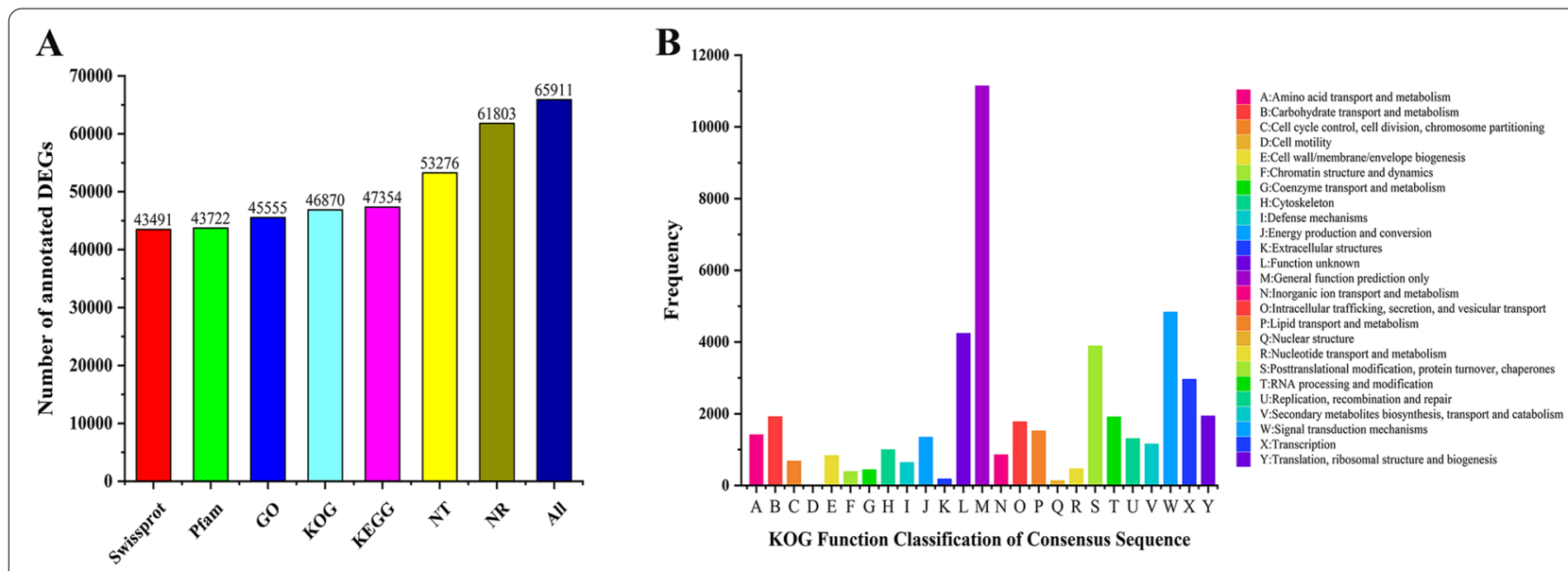

C
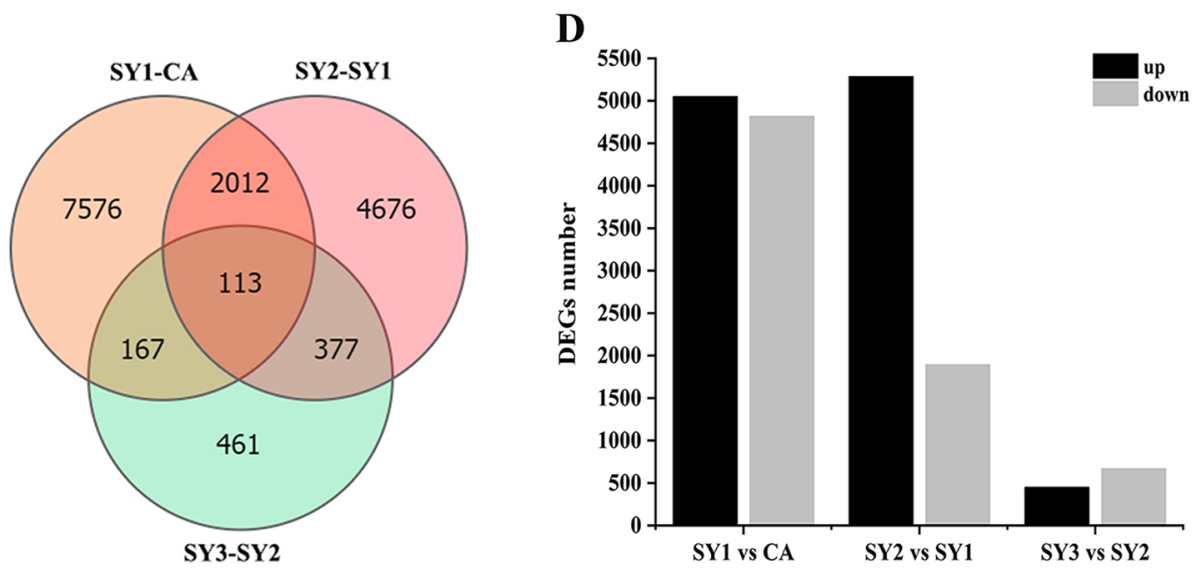

Fig. 4 Statistical analysis of the annotated and differetially expressed unigenes (DEGs) during C. appendiculata symbiotic seed germination. A. Numbers of unigenes annotated into Swissprot, Pfam, GO, KOG, KEGG, NT, NR and total number of annotated unigenes. B. Functional classification of unigenes annotated into KOG. Capital letters A-Y represent the functional categories. C. Venn diagram of all DEGs. D. Up/down-regulated unigenes in all development stages. The total number of DEGs peaked between SY1 and CA. CA, SY1, SY2 and SY3 indicated the symbiotic seeds at 0 day, 6 days, 12 days, and 25 days after sowing,respectively

\section{GO enrichment analysis}

To characterize the DEGs, we performed a GO enrichment analysis using Rage tool. Among the GO terms of DEGs, there were 51, 100 and 153 terms significantly overrepresented in SY1-CA, SY2-SY1 and SY3-SY2, respectively (Table $\mathrm{S} 5$ ). In total, $25 \mathrm{GO}$ terms were over-represented in the three comparisons (Table 3). In particular, these terms included some significantly overrepresented GO terms related to oxidation reactions such as heme binding, monooxygenase activity, dioxygenase activity, peroxidase activity and iron ion binding. In addition, the terms of response to water, metal iron binding and endo-1,4- $\beta$-xylanase activity were annotated in SY1 versus CA. The terms of ATP binding, channel activity and cell wall macromolecule catabolic process were identified in SY2-SY1. However, xyloglucan and carbohydrate metabolic process were also found.

\section{KEGG enrichment analysis}

To investigate the differences in metabolic processes at different stages of symbiotic germination in more detail, a KEGG pathway enrichment analysis with the DEGs was conducted, based on the Qvalue $\leq 0.05$ (Table S6, S7). At SY1-CA, the categories flavonoid biosynthesis, plant hormone signal transduction, fatty acid degradation and phenylpropanoid biosynthesis were significantly enriched. The pathways of phenylpropanoid and flavonoid biosynthesis were also dramatically enriched in SY2SY1 and SY3-SY2. Pathways for fatty acid degradation, carbon fixation in photosynthetic organisms, glycosaminoglycan degradation and carbon metabolism were enriched in SY2-SY1. Pathways of protein processing in endoplasmic reticulum, glycosaminoglycan degradation, plant signal transduction and other glycan degradation were significantly enriched between SY3-SY2. Taken together, these findings suggested that the fungus 
Table 3 Significantly overrepresented gene ontology terms during the symbiotic germination of C. appendiculata with C. disseminatus DJF-10

\begin{tabular}{|c|c|c|c|c|}
\hline Code & GO Term & SY1/CA & Q-value SY2/SY1 & SY3/SY2 \\
\hline \multicolumn{5}{|c|}{ Molecular function } \\
\hline - GO:0020037 & Heme binding & $1.53 \mathrm{E}-11$ & 1.17E-09 & 2.19E-14 \\
\hline GO:0004497 & Monooxygenase activity & $1.92 \mathrm{E}-11$ & 8.15E-09 & $9.85 \mathrm{E}-12$ \\
\hline GO:0051213 & Dioxygenase activity & 7.88E-05 & $9.08 \mathrm{E}-05$ & 0.000427 \\
\hline GO:0004601 & Peroxidase activity & 0.000302 & 0.028842 & 2.37E-05 \\
\hline GO:0016758 & Transferase activity, transferring hexosyl groups & 0.000308 & $2.82 \mathrm{E}-06$ & 0.000111 \\
\hline - GO:0016705 & $\begin{array}{l}\text { Oxidoreductase activity, acting on paired donors, with incorporation or reduc- } \\
\text { tion of molecular oxygen }\end{array}$ & 0.000955 & 0.002064 & 5.36E-06 \\
\hline GO:0005506 & Iron ion binding & 0.00119 & 0.034884 & 2.71E-05 \\
\hline GO:0051787 & Misfolded protein binding & 0.002192 & 0.001156 & 2.22E-07 \\
\hline GO:0016747 & Transferase activity, transferring acyl groups other than amino-acyl groups & 0.002288 & 0.003067 & 4.85E-11 \\
\hline GO:0005200 & Structural constituent of cytoskeleton & 0.002973 & 0.012555 & 0.000248 \\
\hline GO:0003700 & DNA-binding transcription factor activity & 0.006013 & 0.005378 & 2.42E-06 \\
\hline GO:0031072 & Heat shock protein binding & 0.005378 & $6.13 \mathrm{E}-05$ & $6.74 \mathrm{E}-15$ \\
\hline GO:0051082 & Unfolded protein binding & 0.029129 & $9.41 \mathrm{E}-11$ & $6.95 \mathrm{E}-38$ \\
\hline GO:0030246 & Carbohydrate binding & 0.046851 & $2.05 E-21$ & $5.90 \mathrm{E}-08$ \\
\hline \multicolumn{5}{|c|}{ Cellular component } \\
\hline GO:0048046 & Apoplast & 3.80E-05 & $8.62 \mathrm{E}-05$ & 2.74E-09 \\
\hline GO:0005576 & Extracellular region & 0.020282 & $2.24 \mathrm{E}-06$ & $8.25 \mathrm{E}-14$ \\
\hline \multicolumn{5}{|l|}{ Biological process } \\
\hline GO:0042744 & Hydrogen peroxide catabolic process & $3.73 \mathrm{E}-06$ & 0.001527 & $1.08 \mathrm{E}-06$ \\
\hline GO:0006979 & Response to oxidative stress & 0.000395 & 0.007245 & 5.89E-10 \\
\hline GO:0006986 & Response to unfolded protein & 0.000395 & 0.007245 & 5.89E-10 \\
\hline GO:0034620 & Cellular response to unfolded protein & 0.001175 & 0.01359 & $1.84 \mathrm{E}-09$ \\
\hline GO:0007017 & Microtubule-based process & 0.001722 & 0.005323 & 0.000165 \\
\hline GO:0051085 & Chaperone cofactor-dependent protein refolding & 0.002231 & 0.003581 & $3.08 \mathrm{E}-10$ \\
\hline GO:0009664 & Plant-type cell wall organization & 0.006583 & 0.000291 & 0.000293 \\
\hline GO:0045490 & Pectin catabolic process & 0.007005 & 0.000102 & 0.013441 \\
\hline GO:0006457 & Protein folding & 0.047891 & 4.43E-09 & $2.36 \mathrm{E}-25$ \\
\hline
\end{tabular}

Round (filled) circles represent the GO terms were overrepresented during mycorrhizal fungus Tulasnella sp. symbioses with Bletilla striata [29]

induced the expression of plant hormone signal transduction pathways which may break the seed dormancy. Moreover, it induced the expression of fatty acid degradation, carbon metabolism and endoplasmic reticulum processing, and hence improves the efficiency of utilization of stored nutrients.

\section{Expression patterns of symbiosis-related genes at each stage} The lignified seed coat forms a hydrophobic barrier to water permeation during germination. The lignin content of the seeds was sharply reduced after symbiosis with the fungus $C$. disseminatus. Once the hydrophobic barrier was removed, the embryo was able to absorb water efficiently. In this study, we paid much attention to terms related to water absorption. Fifteen genes (including $9 \mathrm{CaDHN} 1$ genes, 4 CaXero 1 genes, 1 hypothetical protein, and 1 CaDHN4-like) related to the term response to water were down-regulated (Fig. 5;
Table S8), and remained at low expression levels in SY2 and SY3. Among these genes, the expression of five CaDHN1 genes (CL1194.Contig2_All, CL1194.Contig3_All, CL1194.Contig5_All, Unigene16008_All and Unigene31280_All) decreased from 176.4, 241.7, 311.4, 166.1 and 38.2 in CA to $0.3,0.6,5.4,4.1$ and 0 in SY1, respectively. Two CaXero1 genes (CL1194.Contig6_All and CL1194.Contig7_All) showed high expression levels of 504.1 and 600.1, respectively at CA, but were downregulated to 4.1 and 12.0, respectively in SY1. The expression level of a hypothetical protein (CL1194.Contig1_All) decreased from 229.7 in CA to 2.9 in SY1. The results suggested that these genes regulate the response to fungal stimulation during seed germination of C. appendiculata after symbiosis with the mycorrhizal fungus.

Indeed, plant hormones play a vital role in the process of symbiotic germination. The current study revealed that a large number of DEGs was annotated 


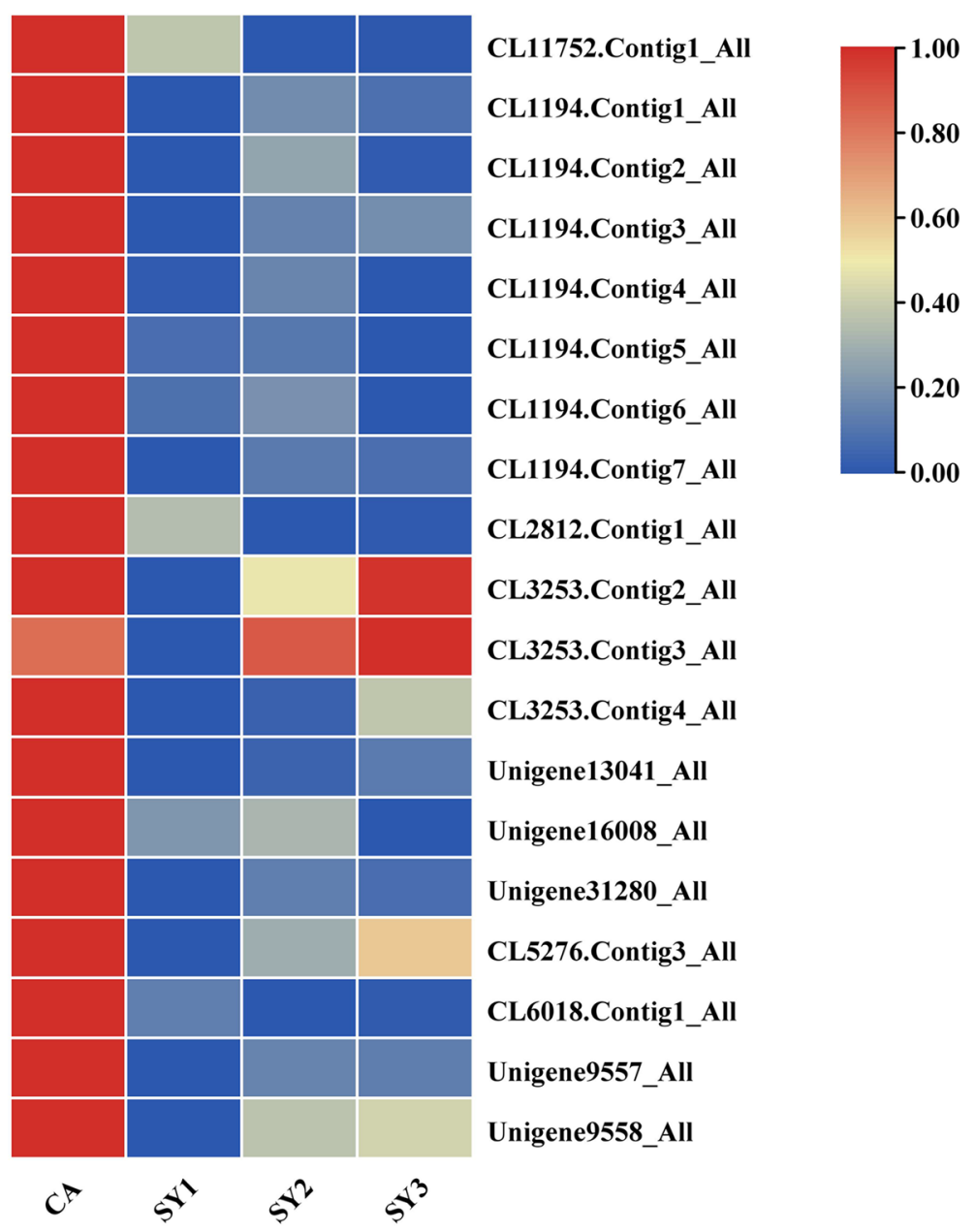

Fig. 5 Cluster heatmap of response to water and ABA biosynthesis term-related gene expression in C. appendiculata during symbiotic germination. CA, SY1, SY2 and SY3 represented symbiotic seeds at 0 day, 6 days, 12 days and 25 days after sowing, respectively

to signal transduction mechanisms. Carotenoid biosynthesis provides the precursors for the synthesis of ABA. In our study, zeaxanthin epoxidase ZEP (CL5276.Contig3_All), 9-cis-epoxycarotenoid dioxygenase NCED3 (CL6018.Contig1_All) and $\beta$-carotene hydroxylase (Unigene9557_All and Unigene9558_All) were significantly down-regulated in SY1-CA (Fig. 5; Table S8). Furthermore, most of the abscisic acid receptor pyrabactin resistance 1-like (PYLs) were upregulated in SY1-CA. Seven genes of protein phosphatase 2C (PP2C), six of SNF1-related protein kinase $2(S n R K 2)$ and four basic region-leucine zipper (bZIP) transcription factors were down-regulated in this group (Fig. 5; Table S8).

\section{Discussion}

The orchid C. appendiculata is one of the most difficult-to-propagate species, a condition which severely restricts its large-scale production. The factors that inhibit C. appendiculata seed germination are still largely unknown. During symbiosis, the lignin content abruptly decreased resulting in a significant increase in water uptake of the seeds compared to the control. These results revealed a negative correlation between lignin and germination, suggesting a possible mechanism whereby the testa-imposed dormancy was broken [34, 42]. The mycorrhizal fungus $C$. disseminatus can degrade lignocellulose by releasing various enzymes such as xylanase, laccase and cellulase $[2,39]$. In the present study, we tested laccase, xylanase and cellulase in the symbiotic 
OMA medium during symbiosis. The results showed that these enzymes were abundant in SY1 and SY2 but decreased at SY3 (Fig. S4) consistently with the physiological data which indicated that lignocellulose was largely degraded at SY1 and SY2. However, the chemical basis of the mechanism involved has not been clearly explained. Moreover, a layer of the seed coat was strained greenish blue with TBO, indicating the presence of polyphenols and lignin in the seed coat of mature seeds, a barrier that disappeared during symbiotic germination [14]. Moreover, the enzymes of cellulases may play an important role in the penetration of fungal hyphae into orchid seeds [35], suggesting that the mycorrhizal fungus $C$. disseminatus essentially scarifies and degrades the lignified seed coat by producing hydrolytic enzymes, resulting in facilitating water uptake and stimulating germination.

Some microorganisms grow on lignocellulose as carbon source and degrade lignin to access cellulose as a preferred carbon source [11,27]. It is noteworthy that $C$. disseminatus is a common species that exists on stumps, buried wood and logs with high wood-decaying capacity [26, 30, 39]. Hynson et al. [17] reported that more than $80 \%$ of the C. appendiculata carbon was obtained from fungal partner, which is more than that in many other orchids. In the present study, the reducing sugar concentration was highest at SY1 in the OMA medium, and decreased thereafter (Fig. S5). The mycelium formed pelotons in the embryo that began to degrade after about 2 weeks. Combing these data with these of enzyme activity, lignocellulose analysis and germination experiment, it seems that $C$. appendiculata was associated with the wood-decaying Coprinellus members $[14,45]$ that obtains nutrients via saprotrophic fungi for seed germination and protocorm development during symbiosis [36, 43]. Fungal invasion appears to trigger metabolic changes in the host plant (especially the nutrient accumulation) during the establishment of symbiosis between $C$. appendiculata and $C$. disseminatus, a process that requires further research.

Four key stages were selected for transcriptome analysis based on the dynamics of fungus penetration into the embryo and key anatomical features in the embryo development. Unfortunately, it was not possible to remove the intracellular fungal hyphae from the symbiotic samples. This meant that the symbiotic samples contained transcripts of $C$. appendiculata and the fungus $C$. disseminatus. Therefore, RNA from the symbiotic fungus library was used for establishing a $C$. disseminatus reference transcriptome and all the mapped reads were driven from the symbiotic samples. This method could be effectively applied to assign genes identified in the transcriptome of plant-fungi associations [47]. The orchid C. appendiculata has been reported to form mycorrhizal associations with saprotrophic fungi [14, 43, 45], which have the potential to elicit strong defense responses in plants. However, our transcriptome data provided no evidence of strong defense activation at the GO terms in C. appendiculata symbiotic samples. This result is similar to those of other symbiotic transcriptome studies on orchids, such as B. strilata [29], Serapias vomeracea [33] and to arbuscular mycorrhizal AM symbiosis. These results suggested that plants recruit a signaling pathway to exploit saprotrophic fungi without inducing stress response [29].

The pathway of plant hormone signal transduction was highly enriched in SY1-CA during $C$. appendiculata symbiotic germination. The data showed that ABA content significantly dropped from $1.38 \mathrm{mgg}^{-1}$ (CA) to $0.08 \mathrm{mgg}^{-1}$ (SY2) with more than 15 -fold reduction, and remained at low level thereafter. During this stage, the genes for key enzymes of ABA synthesis were downexpression. Meanwhile, the genes of $D H N$ family were significantly enriched in SY1-CA, suggesting high expression in CA that effectively alleviate the negative effects of drought stress [37]. During symbiosis, the expression of $D H N$ family was down-regulated. Therefore, we suggest that the low permeability of the seed coat and the high level of $A B A$ in the embryo could act in parallel to hinder the germination of the mature seeds of C. appendiculata.

\section{Conclusion}

In the present study, we investigated the biochemical and transcriptomic changes of $C$. appendiuclata seeds and provided an outline of the important components of the response to symbioses. In summary, the presence of lignified seed coats on the seeds of $C$. appendiculta suggests testa-imposed dormancy that restricts seed germination. As the lignified seed coat was broken down by mycorrhizal fungus $C$. disseminatus, the water absorption by the embryo was improved. The maximal reduction in lignin content was $67.34 \%$ at the first week after sowing. This result was confirmed by FTIR and Py-GC/MS of lignin degradation. Comparative transcriptome profiling revealed the expression patterns of water uptake and ABA biosynthesis candidate genes, which demonstrated that the testa-imposed dormancy was broken. During symbiosis, all genes related to water uptake and ABA biosynthesis were down-regulated. This study extends our understanding of the barriers to seeds germination in $C$. appendiculata. Future work should focus on the molecular mechanistic processes in C. appendiculata seed germination as affected by $C$. disseminatus symbiosis.

\footnotetext{
Abbreviations

C. appendiculata: Cremastra appendiculata; C. disseminatus DJF-10: Coprinellus disseminatus DJF-10; OMA: Oatmeal agar medium; CA: Cremastra appendiculata symbiotic seeds at 0 day after sowing; SY1: Cremastra appendiculata
} 
symbiotic seeds at 6 days after sowing; SY2: Cremastra appendiculata symbiotic seeds at 12 days after sowing; SY3: Cremastra appendiculata symbiotic seeds at 25 days after sowing; Py-GC/MS: Pyrolysis-gas chromatography; FTIR: Fourier transform infrared spectroscopy; GC-MS: Gas Chromatography and Mass Spectrometry; DEG: Differentially expressed genes; NR: Non-redundant proteins; KEGG: Kyoto Encyclopedia of Genes and Genomes; GO: Gene Ontology; FPKM: Fragments per kilobase per million mapped reads; DHN: Dehydrin; ABA: Abscisic acid; ZEP: Zeaxanthin epoxidase; NCED3: 9-cis-epoxyCArotenoid dioxygenases.

\section{Supplementary Information}

The online version contains supplementary material available at https://doi. org/10.1186/s12870-021-03388-6.

Additional file 1: Fig. S1. GC-MS analysis of the chemical compositions of lignocellulose degradation products during symbiotic germination. Fig. S2. NR annotated species distribution of Cremastra appendiculata. Dendrobium catenatum shows the highest similarity. Fig. S3. GO function annotation. The most abundant functions are binding and catalytic activity in terms of molecular function and cellular anatomical entity in terms of cellular component. Fig. S4. Variation of the enzymes during symbioses. Fig. S5. Variation of the reducing sugar concentration produced in the OMA medium during symbioses.

Additional file 2: Table S1. Overview of transcriptome sequence and de novo assembly results. Table S2. Overview of transcriptome assembly showing length of CDS and unigenes. Table S3. Summary of functional annotation of contigs from BLAST searches against public databases. Table S4. DEGs richment ( $\log _{2} F C \geq 2$, Qvalue $\left.\leq 0.05\right)$. Table S5. Significantly overrepresented gene ontology terms during the symbiotic germination of C. appendiculata with C. disseminatus DJF-10. Table S6. Significantly enriched KEGG pathway of different samples. Table S7. DEGs enrichment of KEGG during symbiotic germination. Table S8. DEGs related to water and $A B A$ biosynthesis.

\section{Acknowledgements}

The authors would like to thank Gengrui Lin for assistance with Py-GC/MS experiments, Sijing Peng, Lin Li and Yang Li for assistance with FTIR experiment and plant pollination.

\section{Authors' contributions}

YYG: Methodology, designed, analyzed and wrote the manuscript. MSZ: Designed the research work. JJ and YJZ: Assisted the experiment. MSZ and NXY: Provided critical suggestions for revising the manuscript. All authors read and approved the final manuscript.

\section{Funding}

This work was supported by the National Natural Science Foundation of China (81660627), the Major Special Project of Science and Technology Program in Guizhou (2017-5411-06 and 2017-5788), the National Key Research and Development Program of China (2016YFC0502604), the Construction Project of Modern Industry Technology system of traditional Chinese Medicinal Materials in Cuizhou (GZCYTX-02) and the Construction Program of Biology First-class Discipline in Guizhou (GNYL2017-009).

\section{Availability of data and materials}

The RNA-Seq data has been deposited in the Sequence Read Archive (SRA) at the National Center for Biotechnology Information (NCBI). The accession number is PRJNA762946, which includes 11 accession items (SRX12177800, SRX12177801, SRX12177802, SRX12177803, SRX12177804, SRX12177805, SRX12177806, SRX12177807, SRX12177808, SRX12177809, SRX12177810).

\section{Declarations}

Ethics approval and consent to participate Not applicable.
Consent for publication

Not applicable.

\section{Competing interests}

The authors declare that the research was conducted without any commercial or financial relationship that constructed as a potential conflict of interest.

\section{Author details}

${ }^{1}$ College of Life Sciences, Guizhou University, Guiyang 550025, Guizhou, China. ${ }^{2}$ Key Laboratory of Plant Resources Conservation and Germplasm Innovation in Mountainous Region (Ministry of Education), Guiyang 550025, Guizhou, China.

Received: 8 October 2021 Accepted: 7 December 2021

Published online: 04 January 2022

\section{References}

1. Anders S, Huber W. Differential expression analysis for sequence count data. Genome Biol. 2010;11:106-17.

2. Agnihotri S, Dutt D, Tyagi CH, Kumar A, Upadhyaya JS. Production and biochemical characterization of a novel cellulase-poor alkali-thermotolerant xylanase from Coprinellus disseminatus SW-1 NTCC 1165. World J Microbiol Biotechnol. 2010;26(8):1349-59.

3. Barsberg S, Rasmussen HN, Kodahl N. Composition of Cypripedium calceolus (Orchidaceae) seeds analyzed by attenuated total reflectance IR spectroscopy: in search of understanding longevity in the ground. Am J Bot. 2013;100(10):2066-73.

4. Barsberg ST, Lee YI, Rasmussen HN. Development of C-lignin with G/Slignin and lipids in orchid seed coats-an unexpected diversity exposed by ATR-FT-IR spectroscopy. Seed Sci Res. 2018;28(1):41-51.

5. Baskin CC. Breaking physical dormancy in seeds-focussing on the lens. New Phytol. 2003;158:227-38.

6. Cameron DD, Leake JR, Read DJ. Mutualistic mycorrhiza in orchids: evidence from plant-fungus carbon and nitrogen transfers in the greenleaved terrestrial orchid Goodyera repens. New Phytol. 2006;171(2):405-16.

7. Cao Y, Liang L, Cheng B, Dong Y, Wei J, Tian X, et al. Pretreatment with $\mathrm{NaCl}$ promotes the seed germination of white clover by affecting endogenous phytohormones, metabolic regulation, and dehydrinencoded genes expression under water stress. Int J Mol Sci. 2018;19:3570-84.

8. Chen F, Tobimatsu Y, Havkin-Frenkel D, Dixon RA, Ralph J. A polymer of caffeyl alcohol in plant seeds. Proc Natl Acad Sci U S A. 2012;109(5):1772-7.

9. Christenhusz MJM, Byng JW. The number of known plant species in the world and its annual increase. Phytotaxa. 2016;261:201-17.

10. Delahaie J, Hundertmark M, Bove J, Leprince O, Rogniaux H, Buitink J. LEA polypeptide profiling recalcitrant and orthodox legume seeds reveals ABI3-regulated LEA protein abundance linked to desiccation tolerance. J Exp Bot. 2013;64:4559-73.

11. Dicko M, Ferrari R, Tangthirasunun N, Gautier V, Lalanne C, Lamari F, et al. Lignin degradation and its use in signaling development by the coprophilous ascomycete Podospora anserina. J Fungi. 2020;6(4):278-300.

12. Fang $L, X u X, L i J$, Zheng F, Li M, Yan J, et al. Transcriptome analysis provides insights into the non-methylated lignin synthesis in Paphiopedilum armeniacum seed. BMC Genomics. 2020;21(1):524-38.

13. Finkelstein RR, Gampala SS, Rock CD. Abscisic acid signaling in seeds and seedlings. Plant Cell. 2002;14:S15-45.

14. Gao YY, Peng SJ, Hang Y, Xie GF, Ji N, Zhang MS. Mycorrhizal fungus Coprinellus disseminatus influences seed germination of the terrestrial orchid Cremastra appendicualta (D Don). Makino Sci Hortic. 2021. https:// doi.org/10.1016/j.scienta.2021.110724.

15. Grabherr MG, Haas BJ, Yassour M, Levin JZ, Thompson DA, Amit I, et al. Full-length transcriptome assembly from RNA-Seq data without a reference genome. Nat Biotechnol. 2011;29(7):644-52.

16. Guo $X Y$, Zhang $L$, Wang $X$, Wang $X Z$, Zhang $M H$, Xi YX, et al. Overexpression of Saussurea involucrata dehydrin gene SiDHN promotes cold and drought tolerance in transgenic tomato plants. PLoS One. 2019;14:e0225090.

17. Hynson NA, Schiebold JMI, Gebauer G. Plant family identify distinguishes patterns of carbon and nitrogen stable isotope abundance and nitrogen 
concentration in mycoheterotrophic plants associated with ectmycorrhizal fungi. Ann Bot. 2016;1 18:467-79.

18. Kuga Y, Sakamoto N, Yurimoto H. Stable isotope cellular imaging reveals that both live and degenerating fungal pelotons transfer carbon and nitrogen to orchid protocorms. New Phytol. 2014;202(2):594-605.

19. Langmead B, Salzberg S. Fast gapped-read alignment with bowtie 2. Nat Methods. 2012;9:357-9.

20. Lee Yl, Lee N. Embryo development of Cypripedium formosanum in relation to seed germination in vitro. J Amer Soc Hort Sci. 2005;130(5):747-53.

21. Li B, Dewey CN. RSEM: accurate transcript quantification from RNASeq data with or without a reference genome. BMC Bioinform. 2011:12:323-38.

22. Liu L, Li J, Zeng KW, Jiang Y, Tu PF. Five new biphenanthrenes from Cremastra appendiculata. Molecules. 2016;21 (8):1089-98.

23. Liu SS, Chen J, Li SC, Zeng X, Meng ZX, Guo SX. Comparative transcriptome analysis of genes involved in GA-GID1-DELLA regulatory module in symbiotic and asymbiotic seed germination of Anoectochilus roxburghii (wall.) Lindl. (Orchidaceae). Int J Mol Sci. 2015;16(12):30190-203.

24. Llanes A, Andrade A, Masciarelli O, Alemano S, Luna V. Drought and salinity alter endogenous hormonal profiles at the seed germination phase. Seed Sci Res. 2016;26:1-13.

25. Love MI, Huber W, Anders S. Moderated estimation of fold change and dispersion for RNA-seq data with DESeq2. Genome Biol. 2014;15(12):550-70.

26. Martos F, Dulormne M, Pailler T, Bonfante P, Faccio A, Fournel J, et al. Independent recruitment of saprotrophic fungi as mycorrhizal partners by tropical achlorophyllous orchids. New Phytol. 2009;184:668-81.

27. Mathews SL, Grunden AM, Pawlak J. Degradation of lignicellulose and lignin by Paenibacillus glucanolyticus. Int Biodeterior Biodegradation. 2016;110:79-86

28. Min CW, Lee SH, Cheon YE, et al. In-depth proteomic analysis of Glycine max seeds during controlled deterioration treatment reveal a shift in seed metabolism. J Proteome. 2017;169:125-35.

29. Miura C, Yamaguchi K, Miyahara R, Yamamoto T, Fuji M, Yagame T, et al. The mycoheterotrophic symbiosis between orchids and mycorrhizal fungi possesses major components shared with mutualistic plant-mycorrhizal symbioses. Mol Plant-Microbe Interact. 2018;31(10):1032-47.

30. Oliver JP, Perkins J, Jellison J. Effect of fungal pretreatment of wood on successional decay by several inky cap mushroom species. Int Biodeterior Biodegradation. 2010;7:646-51.

31. Paulsen TR, Colville L, Kranner I, Daws MI, Högstedt G, Vandvik V, et al. Physical dormancy in seeds: a game of hide and seek? New Physiol. 2013;198:496-503.

32. Perez J, Munon-Dorado J, de la Rubia T, Martinez J. Biodegradation and biological treatments of cellulose-hemicellulise and lignin: an overview. Int Microbiol. 2002;5:53-63.

33. Perotto S, Rodda M, Benetti A, Sillo F, Ercole E, Rodda M, et al. Gene expression in mycorrhizal orchid protocorms suggests a friendly plantfungus relationship. Planta. 2014;239(6):1337-49.

34. Pierce S, Spada A, Caporali E, Ceriani RM, Buffa G. Enzymatic scarification of Anacamptis morio (Orchidaceae) seed facilitates lignin degradation, water uptake and germination. Plant Biol. 2019;21:409-14.

35. Rasmussen HN. Terrestrial orchids, from seed to mycotrophic plant. Cambridge: Cambridge University Press; 1995.

36. Rasmussen HN, Dixon KW, Jersakova J, Tesitelova T. Germination and seedling establishment in orchids: a complex of requirements. Ann Bot. 2015;116(3):391-402.

37. Riyazuddin R, Nisha N, Singh K, Verma R, Gupta R. Involvement of dehydrin proteins in mitigating the negative effects of drought stress in plant. Plant Cell Rep. 2021. https://doi.org/10.1007/s00299-021-02720-6.

38. Shim JS, Kim JH, Lee J, Kim SN, Kwon HJ. Anti-angiogenic activity of a homoisoflavanone from Cremastra appendiculata. Planta Med. 2004;70(2):171-3.

39. Singh S, Tyagi CH, Dutt D, Upadhyaya JS. Production of high level of celIulase-poor xylanases by wild strains of white-rot fungus Coprinellus disseminatus in soild-state fermentation. New Biotechnol. 2009;26:165-70.

40. Smith SE. Physiology and ecology of orchid mycorrhizal fungi with reference to seedling nutrition. New Physiol. 1966. https://doi.org/10.1111/j. 1469-8137.1966.tb05972.x.
41. State pharmacopoeia committee. Pharmacopoeia of the People's Republic of China, vol. I. Beijing: China Pharmaceutical Science and Technology Press; 2020

42. Steinbrecher T, Leubner-Metzger G. Tissue and cellular mechanics of seeds. Curr Opin Genet Dev. 2018:51:1-10.

43. Suetsugu K, Haraguchi TF, Tayasu I. Novel mycorrhizal cheating in a green orchid: Cremastra appendiculata depends on carbon from deadwood through fungal associations. New Phytol. 2021. https://doi.org/10.1111/ nph.17313.

44. Vasudevan $\mathrm{R}$, van Staden J. Fruit harvesting time and corresponding morphological changes of seed integuments influence in vitro seed germination of Dendrobium nobile Lindl. Plant Growth Regul. 2009;60(3):237-46.

45. Yagame T, Funabiki E, Nagasawa E, Fukiharu T, Iwase K. Identification and symbiotic ability of Psathyrellaceae fungi isolated from a photosynthetic orchid, Cremastra appendiculata (Orchidaceae). Am J Bot. 2013;100(9):1823-30.

46. Zang H, Du X, Wang J, Cheng Y, Wang Y, Sun S, et al. Insight into coldactive xylanase production and xylan degradation pathways in psychrotrophic Acinetobacter sp. HC4 from the cold region of China. Cellulose. 2020;27(13):7575-89.

47. Zeng X, Li Y, Ling H, Liu S, Liu M, Chen J, et al. Transcriptomic analyses reveal clathrin-mediated endocytosis involved in symbiotic seed germination of Gastrodia elata. Bot Stud. 2017;58(1):31-41.

48. Zhang H, He D, Yu J, Li M, Damaris RN, Gupta R, et al. Analysis of dynamic protein carbonylation in rice embryo during germination throught APSWATH. Proteomics. 2016;16:989-1000.

49. Zhou W, Chen F, Luo X, Dai Y, Yang Y, Zheng C, et al. A matter of life and death: molecular, physiological, and environment regulatin of seed longevity. Plant Cell Environ. 2020;43:293-302.

\section{Publisher's Note}

Springer Nature remains neutral with regard to jurisdictional claims in published maps and institutional affiliations.

Ready to submit your research? Choose BMC and benefit from:

- fast, convenient online submission

- thorough peer review by experienced researchers in your field

- rapid publication on acceptance

- support for research data, including large and complex data types

- gold Open Access which fosters wider collaboration and increased citations

- maximum visibility for your research: over 100M website views per year

At BMC, research is always in progress.

Learn more biomedcentral.com/submissions 